\title{
Nonlinear effects in propagation of long-range surface plasmon polaritons in gold strip waveguides
}

\author{
Lysenko, Oleg; Bache, Morten; Malureanu, Radu; Lavrinenko, Andrei
}

Published in:

Proceedings of SPIE

Link to article, DOI:

$10.1117 / 12.2224500$

Publication date:

2016

Document Version

Publisher's PDF, also known as Version of record

Link back to DTU Orbit

Citation (APA):

Lysenko, O., Bache, M., Malureanu, R., \& Lavrinenko, A. (2016). Nonlinear effects in propagation of long-range surface plasmon polaritons in gold strip waveguides. In Proceedings of SPIE (Vol. 9884). [988420] SPIE International Society for Optical Engineering. Proceedings of SPIE - The International Society for Optical Engineering https://doi.org/10.1117/12.2224500

\section{General rights}

Copyright and moral rights for the publications made accessible in the public portal are retained by the authors and/or other copyright owners and it is a condition of accessing publications that users recognise and abide by the legal requirements associated with these rights.

- Users may download and print one copy of any publication from the public portal for the purpose of private study or research.

- You may not further distribute the material or use it for any profit-making activity or commercial gain

- You may freely distribute the URL identifying the publication in the public portal 


\title{
Nonlinear effects in propagation of long-range surface plasmon polaritons in gold strip waveguides
}

\author{
Oleg Lysenko*, Morten Bache, Radu Malureanu, Andrei Lavrinenko \\ Department of Photonics Engineering, Technical University of Denmark, \\ Oersteds Plads, Building 345 V, Kongens Lyngby 2800, Denmark
}

\begin{abstract}
This paper is devoted to experimental and theoretical studies of nonlinear propagation of a long-range surface plasmon polariton (LRSPP) in gold strip waveguides. The plasmonic waveguides are fabricated in house, and contain a gold layer, tantalum pentoxide adhesion layers, and silicon dioxide cladding. The optical characterization was performed using a high power picosecond laser at $1064 \mathrm{~nm}$. The experiments reveal two nonlinear optical effects: nonlinear power transmission and spectral broadening of the LRSPP mode in the waveguides. Both nonlinear optical effects depend on the gold layer thickness. The theoretical model of these effects is based on the third-order susceptibility of the constituent materials. The linear and nonlinear parameters of the LRSPP mode are obtained, and the nonlinear Schrödinger equation is solved. The dispersion length is much larger than the waveguides length, and the chromatic dispersion does not affect the propagation of the plasmonic mode. We find that the third-order susceptibility of the gold layer has a dominant contribution to the effective third-order susceptibility of the LRSPP mode. The real part of the effective third-order susceptibility leads to the observed spectral broadening through the self-phase modulation effect, and its imaginary part determines the nonlinear absorption parameter and leads to the observed nonlinear power transmission. The experimental values of the third-order susceptibility of the gold layers are obtained. They indicate an effective enhancement of the thirdorder susceptibility for the gold layers, comparing to the bulk gold values. This enhancement is explained in terms of the change of the electrons motion.
\end{abstract}

Keywords: Plasmonics, nonlinear waveguides, ultrafast nonlinear optics, surface plasmon polaritons

\section{INTRODUCTION}

Plasmonic waveguides have unique optical properties, and found applications in many areas, such as components of integrated optics [1], nanofocusing of electromagnetic radiation [2], sensors [3], and photodetectors [4]. Meanwhile, nonlinear optical properties of plasmonic waveguides (with one or several metal-dielectric interfaces) are investigated much less. This problem is tricky, because the nonlinear metal properties are not yet well understood. One of the possible theoretical approaches here is based on the hydrodynamic model for the free-electron gas [5]. In other works, the nonlinear propagation of the LRSPP modes in plasmonic waveguides is studied in terms of either the cascaded secondorder nonlinearity at a metal-dielectric interface [6] or the third-order nonlinearity of metal and dielectric in plasmonic nanorod structures [7]. In the latter case, the third-order nonlinearity for gold is obtained from the two-temperature model of electrons dynamics [8] and the ideal lossless metal is assumed [7]. An overview of some theoretical and experimental results related to the third-order susceptibility of gold has been published recently by Boyd et al. [9]. The enhancement of nonlinear properties can even overcome the constituent material's constrains if the metamaterials concept is involved [10].

Recent experiments confirm the presence of nonlinear propagation effects in plasmonic waveguides, much like the ones that for decades have been fueling nonlinear fiber optics [11]. We recently conducted an experiment [12] using the same system used in the present paper, namely a strip plasmonic gold waveguide, and we observed that the loss performance was nonlinear as the power was increased, which we attributed to the imaginary part of the nonlinearity of gold, and we also saw indications of self-phase modulation (SPM) in the spectrum. Both effects were significantly affected by the nanometer-scale thickness of the gold layer. To our knowledge such a study has not yet been done in plasmonic waveguides; an early study [13] used instead cw-like pumping and obtained significant thermal contributions to the nonlinear phase. In another recent experiment Baron et al. [14] measured the imaginary component of the third-order

*ollyse@fotonik.dtu.dk

Nanophotonics VI, edited by David L. Andrews, Jean-Michel Nunzi, Andreas Ostendorf, Proc. of SPIE Vol. 9884, $988420 \cdot$ (c) 2016 SPIE · CCC code: 0277-786X/16/\$18 · doi: 10.1117/12.2224500 
susceptibility for bulk gold by studying nonlinear propagation of surface-plasmon polaritons on a single air/metal interface.

In our experiment, the fabricated plasmonic strip waveguides consist of a gold core, tantalum pentoxide adhesion layers, and silicon dioxide cladding. This configuration provides effective propagation of the LRSPP mode [15]. We take into account that all constituent materials (metal, adhesion layer, and cladding) exhibit a nonlinear response and attribute an effective nonlinearity of the LRSPP waveguide mode, and for the metal part of the waveguide the linear and nonlinear losses are considered as well. This effective nonlinearity of the LRSPP mode leads to two interesting nonlinear effects. The first effect is a nonlinear saturation of the average power of the plasmonic mode. The second effect is a nonlinear phase modulation with spectral broadening of the LRSPP mode. Our theoretical model solves the nonlinear Schrödinger equation for the LRSPP mode, and confirms the observed nonlinear effects.

\section{FABRICATION PROCESS}

The samples with waveguides were fabricated in house, according to the following procedure. A silicon wafer with a thick $(\sim 6.5 \mu \mathrm{m})$ layer of $\mathrm{SiO}_{2}$ on top was used as a substrate. A standard ultraviolet lithography process was applied to pattern a photoresist layer. Then, a gold layer and adhesion layers were deposited using a sputtering machine (Lesker). A chemical solution (Piranha) was used to remove the photoresist layer afterwards. The waveguides profile contained four layers: a bottom $\mathrm{Ta}_{2} \mathrm{O}_{5}$ layer, $\mathrm{Au}$ layer, top $\mathrm{Ta}_{2} \mathrm{O}_{5}$ layer, and small $\mathrm{SiO}_{2}$ layer [12]. The layers were deposited one after another in one sputtering program, and the known deposition times and rates were used to calculate the thickness values. It is well-known that the metal layer roughness increases the propagation losses of the LRSPP mode [16]. The atomic force microscope (AFM) Dimension Icon (Bruker) was used to measure the gold layers roughness (Fig. 1a). The roughness profile of the gold surface shows the spikes with the maximum height values $\sim 2 \mathrm{~nm}$. The root mean square (RMS) value for the roughness of the deposited gold layers is $0.67 \mathrm{~nm}$. Several measurements of $2 \mu \mathrm{m}$ regions at arbitrary places of the gold surface were performed and confirmed the reported RMS roughness.

To verify the quality of the deposited metal and adhesion layers, a separate test wafer was prepared. A silicon wafer with a thick $(\sim 800 \mathrm{~nm})$ layer of silicon dioxide was used as a substrate. A gold layer and an adhesion layer were deposited on top by the same procedure, but in different parts of the wafer. Their surfaces were imaged by the scanning electron microscope (SEM) SUPRA (Carl Zeiss). The gold surface (Fig. 1b) has many bright dots related to the roughness spikes. The imaging showed that the both layers are continuous and of high quality.

After the sputtering of the waveguides, a thick $(\sim 5.5 \mu \mathrm{m})$ cladding layer of $\mathrm{SiO}_{2}$ was deposited on top using a standard plasma-enhanced chemical vapor deposition method. The cladding thickness and linear refractive index were measured by a multiple-angle reflectometer (FilmTek 4000). The refractive index value for deposited silicon dioxide is 1.456 at the wavelength $1064 \mathrm{~nm}$. Overall, three wafers with the gold layer thicknesses $t=22,27$, and $35 \mathrm{~nm}$ were fabricated by the same procedure and used in the experiment. The ready wafers were diced and several samples with lengths 2, 3, and 4 $\mathrm{mm}$ were selected for optical characterization. Each sample contained five identical waveguides with $10 \mu \mathrm{m}$ width, and $100 \mu \mathrm{m}$ spacing. Thus, the waveguides did not interfere with each other and could be investigated independently.

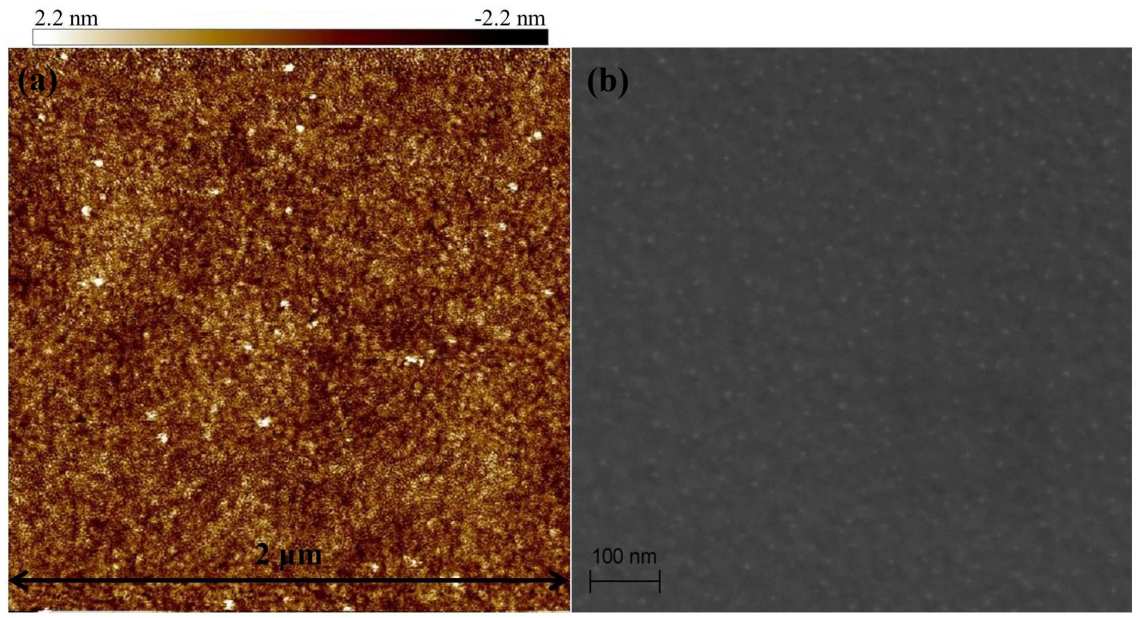

Figure 1. (a) AFM measurement of the gold layers roughness and (b) SEM image of the gold surface. 


\section{EXPERIMENTAL RESULTS}

\subsection{Experimental setup}

The experimental setup is shown in Fig. 2. The laser source was the picosecond pump source for the SuperK EXTREME (NKT Photonics) with the average output power tunable in the range $0-15 \mathrm{~W}$, repetition rate $78 \mathrm{MHz}$, pulse duration $\sim 3$ ps, and peak wavelength $1064 \mathrm{~nm}$. The free-space laser beam was linearly polarized by a polarizer (Thorlabs), shaped by a Gaussian filter (10LF04-1064, FWHM $3.5 \mathrm{~nm}$, Newport), attenuated by a set of neutral-density (ND) attenuation filters, and delivered to the sample by a polarization-maintaining fiber (LMA-PM-10, NKT Photonics). The laser beam polarization was aligned to match the fast axis of the polarization-maintaining fiber and to match the transverse magnetic polarization of the LRSPP mode in plasmonic waveguides. The end-fire coupling method was used to excite plasmonic modes [17]. A single mode fiber (LMA-10, NKT Photonics) collected and delivered the transmitted optical flux to the optical spectrum analyzer (OSA) AQ6315E (Yokogawa). The end-fire coupling alignment was controlled by an optical microscope. Before the transmission measurements, the LRSPP mode in each waveguide was imaged by a lens (Newport) and camera beam profiler (Thorlabs), and the transverse magnetic polarization of the plasmonic mode was verified by the linear polarizer [18]. This polarization check verified coupling to the LRSPP mode in each plasmonic waveguide.

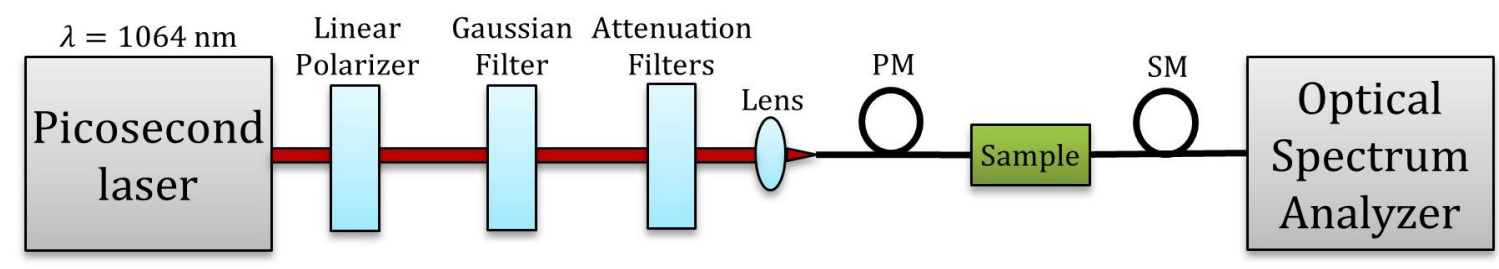

Figure 2. Experimental setup for nonlinear optical characterization.

\subsection{Linear characterization}

Firstly, the linear characterization of the waveguides was performed. The laser was tuned to a low average power $(<1$ $\mathrm{mW}$ ), and the transmitted power was measured by the OSA for waveguides with different lengths and metal thicknesses. The insertion loss graphs were used to calculate propagation loss $\alpha$ per unit length and coupling loss $C$ per two facets for the plasmonic waveguides with the metal thickness values 22, 27, and $35 \mathrm{~nm}$ (Fig. 3). For each value of the length and thickness the measurements of five identical waveguides were averaged, and the linear fit was calculated by the least squares method. The coupling loss was obtained as the intersection of the fitted lines with the vertical axis. The propagation loss was calculated as the slope angle to the fitting lines. The calculated values for $\alpha$ and $C$, with the calculation errors are presented in Table 1. The obtained experimental values show that the linear propagation loss per unit length increases in $\sim 1.8$ times as the gold layer thickness increases from $22 \mathrm{~nm}$ to $35 \mathrm{~nm}$. The coupling loss per two facets varies by $\sim 10 \%$, and is completely within the calculation errors. This conclusion is in accordance with the previous measurements of the similar plasmonic waveguides [19]. The obtained values for $\alpha$ are $\sim 2-3$ times higher than the results published by Berini et al., and several possible reasons explain this. First, the maximum values of the roughness spikes of the gold layers are $\sim 2 \mathrm{~nm}$ in comparison with $\sim 1 \mathrm{~nm}$ in Ref. [19]. Second, the peak wavelength 1550 $\mathrm{nm}$ used in Ref. [19] provides lower attenuation of the LRSPP mode in plasmonic waveguides than the peak wavelength $1064 \mathrm{~nm}[20]$.

Table 1. Experimental values of the propagation and coupling loss for plasmonic waveguides with different thicknesses.

\begin{tabular}{|c|c|c|}
\hline $\boldsymbol{t}(\mathbf{n m})$ & $\alpha(\mathbf{d B} / \mathbf{m m})$ & $\boldsymbol{C}(\mathbf{d B})$ \\
\hline 22 & $7.8 \pm 0.2$ & $3.2 \pm 0.5$ \\
\hline 27 & $11.1 \pm 0.2$ & $3.3 \pm 0.8$ \\
\hline 35 & $13.8 \pm 0.3$ & $3.5 \pm 1.0$ \\
\hline
\end{tabular}




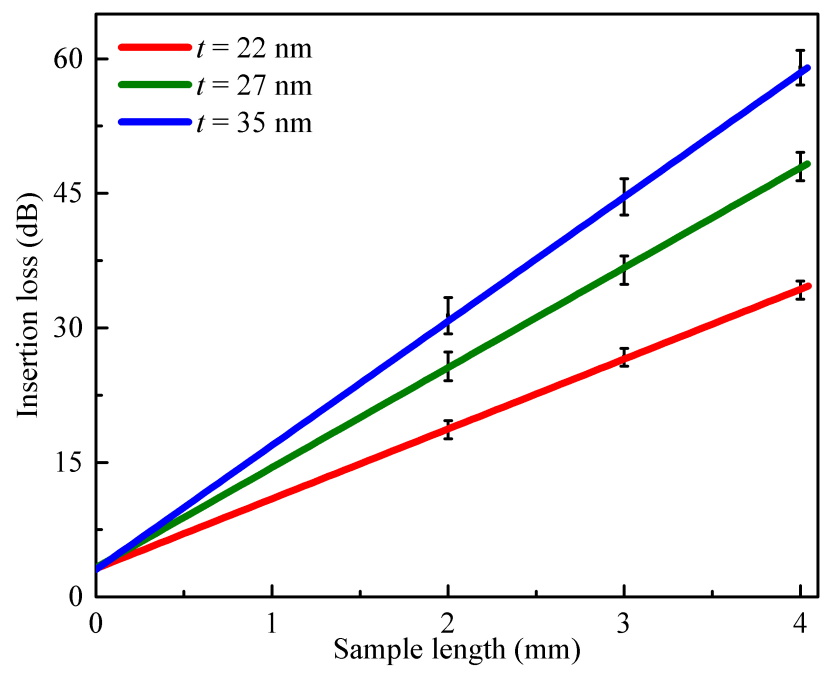

Figure 3. Propagation loss and coupling loss measurements of plasmonic waveguides with different gold layer thicknesses.

\subsection{Nonlinear power measurements}

Then, the nonlinear characterization of the plasmonic waveguides was performed. In order to obtain the necessary amount of measurements, the input power was varied by either changing the ND attenuation filters or the output power from the laser. The transmitted power was measured by the OSA for the waveguides of length $3 \mathrm{~mm}$ and metal thicknesses 22, 27, and $35 \mathrm{~nm}$. The normalized obtained dependencies of the transmitted power $P_{\text {out }}$ with respect to the delivered power $P_{\text {in }}$ have several interesting features (Fig. 4). The first feature is the nonlinear trends of dependencies $P_{\text {out }}\left(P_{\text {in }}\right)$ at high values of the input power $P_{\text {in }}$. The second feature is that these nonlinear trends clearly depend on the metal layers thickness. The plasmonic waveguides with thinner metal layers have more pronounced relative deviation from the linear power dependences at the same values of the input power. For each value of the metal thickness the measurements of five identical waveguides are averaged and the nonlinear curve fit was calculated as

$$
P_{\text {out }}=a P_{\text {in }}-b P_{\text {in }}^{2} \text {, }
$$

where $a$ and $b$ are the fitting coefficients. Here the input and output powers are corrected for the coupling loss at the end facets. The calculated values for $a$, and $b$, with the calculation errors are presented in Table 2. The coefficient $a$ is related to the linear loss parameter $\alpha$ of the LRSPP mode as $a=e^{-\alpha l}$, where $l$ is the waveguide length. The calculated values of $\alpha$ are $8.82 \mathrm{~dB} / \mathrm{mm}, 12.0 \mathrm{~dB} / \mathrm{mm}$, and $17.3 \mathrm{~dB} / \mathrm{mm}$ for the metal thicknesses 22,27 , and $35 \mathrm{~nm}$, accordingly. These values are somewhat higher than the values of linear losses at low power characterization, and the origin of this is at present unclear. The coefficient $b$ is related to nonlinear absorption coefficient $\beta$ of the plasmonic mode. The nonlinearities of the constituent materials (metal, adhesion, and cladding) in plasmonic waveguides, as well as heating for high powers, contribute to the effective nonlinearity of the LRSPP mode.

Table 2. Fit coefficients $a$ and $b$ for the plasmonic waveguides with different thicknesses.

\begin{tabular}{|c|c|c|}
\hline $\boldsymbol{t}(\mathbf{n m})$ & $\boldsymbol{a}$ & $\boldsymbol{b}(\mathbf{1} / \mathbf{W})$ \\
\hline 22 & $(2.26 \pm 0.01) \cdot 10^{-3}$ & $(1.54 \pm 0.02) \cdot 10^{-3}$ \\
\hline 27 & $(2.49 \pm 0.01) \cdot 10^{-4}$ & $(1.38 \pm 0.02) \cdot 10^{-4}$ \\
\hline 35 & $(6.53 \pm 0.03) \cdot 10^{-6}$ & $(3.09 \pm 0.08) \cdot 10^{-6}$ \\
\hline
\end{tabular}




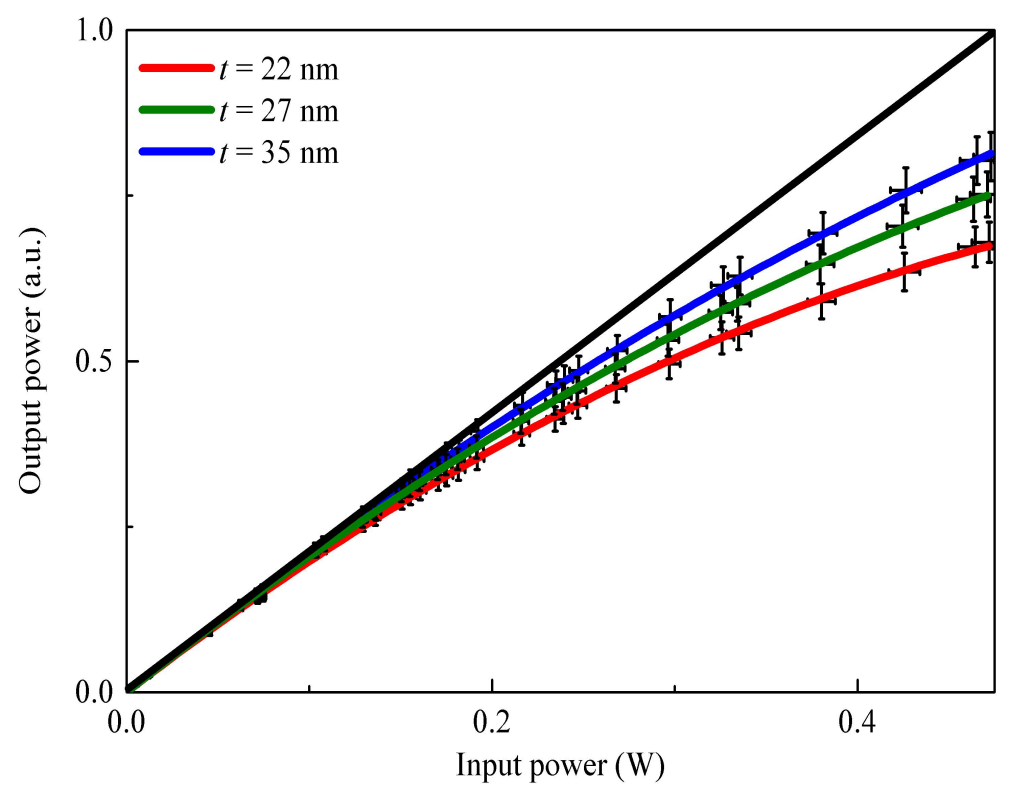

Figure 4. Nonlinear power transmission of plasmonic waveguides with different gold layer thicknesses.

In terms of the nonlinear response of the constituent materials, gold has a dominant contribution to the effective thirdorder susceptibility $\chi_{\text {eff }}^{(3)}$ of the plasmonic mode [21]. The effective third-order susceptibility of the LRSPP mode is related to the nonlinear absorption coefficient $\beta$ as follows [22]:

$$
\beta=\frac{\beta_{\mathrm{c}}}{A_{\mathrm{eff}}}=\frac{3 \omega}{2 \varepsilon_{0} c^{2} n_{0}^{2} A_{\mathrm{eff}}} \operatorname{Im}\left[\chi_{\mathrm{eff}}^{(3)}\right],
$$

Here $\beta_{\mathrm{c}}$ is the conventional definition of the nonlinear absorption coefficient [22], $\omega=2 \pi c / \lambda$ is the light angular frequency, $c$ is the speed of light in vacuum, $\varepsilon_{0}$ is the vacuum permittivity, $n_{0}$ is the effective linear refractive index of the LRSPP mode, and $A_{\text {eff }}$ is the effective area of the LRSPP mode. The nonlinear coefficient $b$ depends on both the nonlinear parameters of the LRSPP mode and the laser parameters. By considering the pulse propagation equation [11], it is straightforward to show that the ratio $b / a \approx \beta /\left(\alpha T f_{\text {rep }}\right)$, where $T$ is the laser pulse duration, and $f_{\text {rep }}$ is the laser repetition rate. We analyzed the structure of our plasmonic waveguides, where the total effective nonlinearity has contributions from all layers, calculated through the overlap integral of the spatial distribution of the plasmonic mode in each layer and using the respective bulk nonlinearity of each layer. The values of the field integrals from the gold core $\theta_{\mathrm{Au}}$ are $\sim 1.1 \cdot 10^{-4}, \sim 1.4 \cdot 10^{-4}$, and $\sim 1.9 \cdot 10^{-4}$ for the layers thickness 22,27 , and $35 \mathrm{~nm}$ correspondingly. And the values of the effective area of the waveguide are $\sim 11.2 \mu \mathrm{m}^{2}, \sim 10.2 \mu \mathrm{m}^{2}$, and $\sim 9.2 \mu \mathrm{m}^{2}$ correspondingly. Through this analysis, which will be presented in detail elsewhere, we found that gold has by far the dominating contribution to the total effective nonlinearity: the contribution from the gold layer is two orders of magnitude bigger $\left(\sim 10^{-20} \mathrm{~m}^{2} / \mathrm{V}^{2}\right)$ than the one from the tantalum pentoxide layer $\left(\sim 10^{-22} \mathrm{~m}^{2} / \mathrm{V}^{2}\right)$, although the latter has a quite large cubic nonlinearity [23]. The calculated value of the real part of the effective refractive index of the plasmonic mode $\operatorname{Re}\left[n_{\mathrm{eff}}\right] \approx 1.47$ is much larger than the value of its imaginary part $\operatorname{Im}\left[n_{\mathrm{eff}}\right] \approx 0.01$. Therefore we can take as a good approximation the following formula for the nonlinear absorption coefficient $\beta \approx\left(3 \omega \theta_{\mathrm{Au}} \operatorname{Im}\left[\chi_{\mathrm{Au}}^{(3)}\right]\right) /\left(2 \varepsilon_{0} c^{2} n_{0}^{2} A_{\mathrm{eff}}\right)$, where $\chi_{\mathrm{Au}}^{(3)}$ is the third-order susceptibility of the gold layers, and the obtained coefficients $a$ and $b$ are used to extract the imaginary part of $\chi_{\mathrm{Au}}^{(3)}$. 


\subsection{Nonlinear spectral measurements}

The spectra of the transmitted optical flux were measured by the OSA and in the wavelength range 1058-1069 nm. They show several interesting features (Fig. 5). Firstly, spectral broadening and splitting are observed, a clear sign of SPM influenced by two-photon absorption from the real and imaginary part of $\chi^{(3)}$, respectively [11]. This was confirmed with numerical simulations of the nonlinear Schrödinger equation, giving almost identical spectra as those in Fig. 5, where the experimental input pulse was used and no free parameters were adopted (all linear and nonlinear parameters were those calculated by our waveguide analysis or measured experimentally). The second feature is that the spectral broadening and splitting in the output spectra differs slightly for each case. We compared the values of the effective spectral widths for the input pulse and output pulses at the splitting level $(\sim 0.05)$, where for simplicity the side peaks with lower intensities ( 0.01-0.03) were neglected. To do an analytical estimate, we approximate the input pulse with a Gaussian one and calculate the nonlinear spectral broadening factor (SBF) from comparing it to a Gaussian fit to the two distinct SPM peaks. The SBF then relates to the effective nonlinear coefficient $\gamma$ by [11]:

$$
\mathrm{SBF} \approx 0.86 \gamma P_{0} \frac{1-e^{-\alpha l}}{\alpha},
$$

where $\gamma=\left(\omega n_{\text {2eff }}\right) /\left(c A_{\text {eff }}\right), P_{0}$ is the peak power of an input pulse, $n_{2 \text { eff }}$ is the effective intensity dependent refractive of the LRSPP mode, and $l$ is the length of a plasmonic waveguide. This result assumes a purely Gaussian input pulse, which is transform limited (no chirp across the pulse). Although the input pulse in the experiment was not transform limited and its spectrum had multiple modulated peaks, this expression serves as a rough estimate of the effective waveguide nonlinearity $\gamma$ in the sample; such an approach is also justified by the close agreement with the numerical simulations. From the second equation in Eq. (3) and using the relation between $n_{2 \text { eff }}$ and $\operatorname{Re}\left[\chi_{\text {eff }}^{(3)}\right]$ the following formula is realized $\gamma \approx\left(3 \omega \theta_{\mathrm{Au}} \operatorname{Re}\left[\chi_{\mathrm{Au}}^{(3)}\right]\right) /\left(4 \varepsilon_{0} c^{2} n_{0}^{2} A_{\mathrm{eff}}\right)$, since as argued above for the nonlinear loss, the dominating contribution comes from gold. The experimental values of the SBF are used to extract the real part of the third-order susceptibility of the gold layers showing its dependence on the layer thickness.
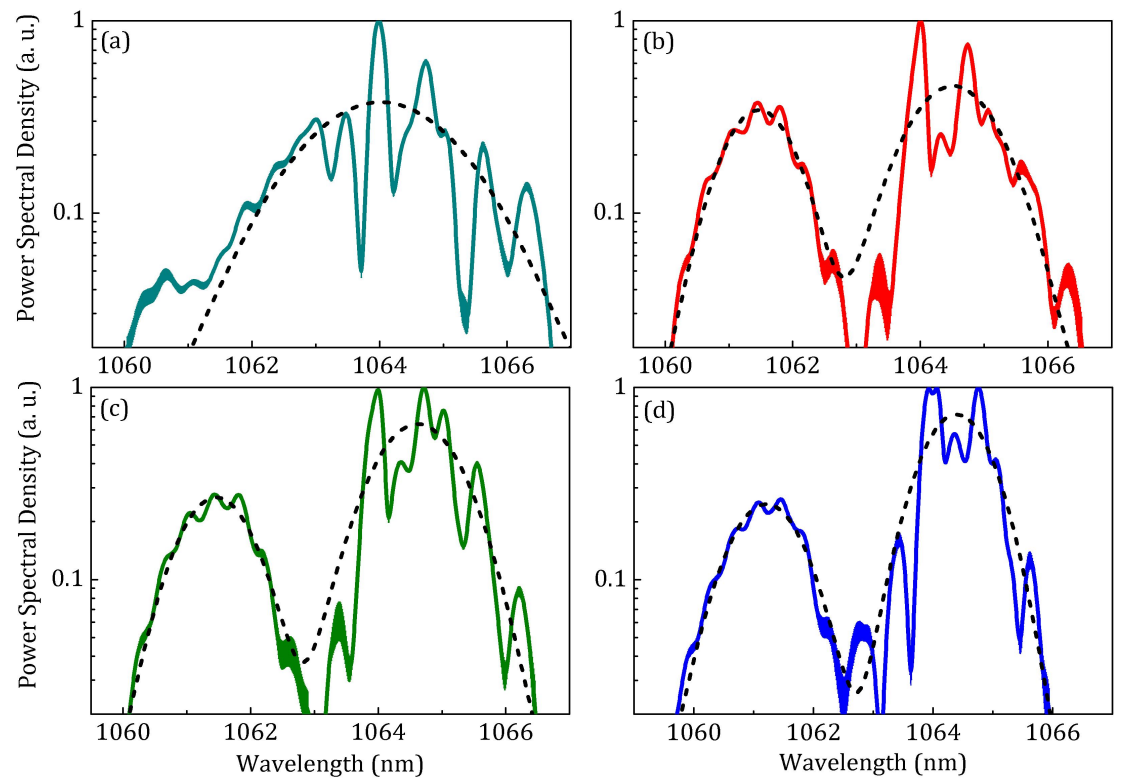

Figure 5. (a) Input pulse spectrum, and output pulse spectra for plasmonic waveguides at the maximum input power ( $480 \mathrm{~mW})$ with the gold layer thickness (b) $t=22 \mathrm{~nm}$, (c) $t=27 \mathrm{~nm}$, and (d) $t=35 \mathrm{~nm}$. Dashed lines show a Gaussian fit to the measured spectra.

\subsection{Third-order nonlinear susceptibility of gold}

The calculated values of the real and imaginary parts of the third-order susceptibility for gold layers with different thicknesses, and the calculation errors are presented in Table 3. Both the real and imaginary parts decrease as the layer 
thickness increases. The experimental values for the third-order susceptibility are plotted in Fig. 6 and the nonlinear curve fit was calculated as follows:

$$
\chi_{\mathrm{Au}}^{(3)}=\chi_{\infty}^{(3)}+\frac{C}{t},
$$

where $\chi_{\infty}^{(3)}$ is the third-order susceptibility for bulk gold [7], $t$ is the gold layer thickness, and $C$ is the experimental coefficient. The fitting $1 / t$ here was done by analogy with the change of the electrons collisions frequency in metal nanoparticles with the radius [24]. The calculated values for the real and imaginary parts of the experimental coefficient $C$ are: $\operatorname{Re}[C]=(5.82 \pm 0.15) \cdot 10^{-24} \mathrm{~m}^{3} / \mathrm{V}^{2}$, and $\operatorname{Im}[C]=(9.34 \pm 1.79) \cdot 10^{-26} \mathrm{~m}^{3} / \mathrm{V}^{2}$. For comparison we plot also the third-order susceptibility for bulk gold $\chi_{\infty}^{(3)}=(4.56+0.48 i) \cdot 10^{-17} \mathrm{~m}^{2} / \mathrm{V}^{2}[7]$. We here again stress that the obtained values should be taken with caution as they were extracted assuming a transform-limited Gaussian input pulse. Further experiments with cleaner pulse profiles should illuminate this issue.
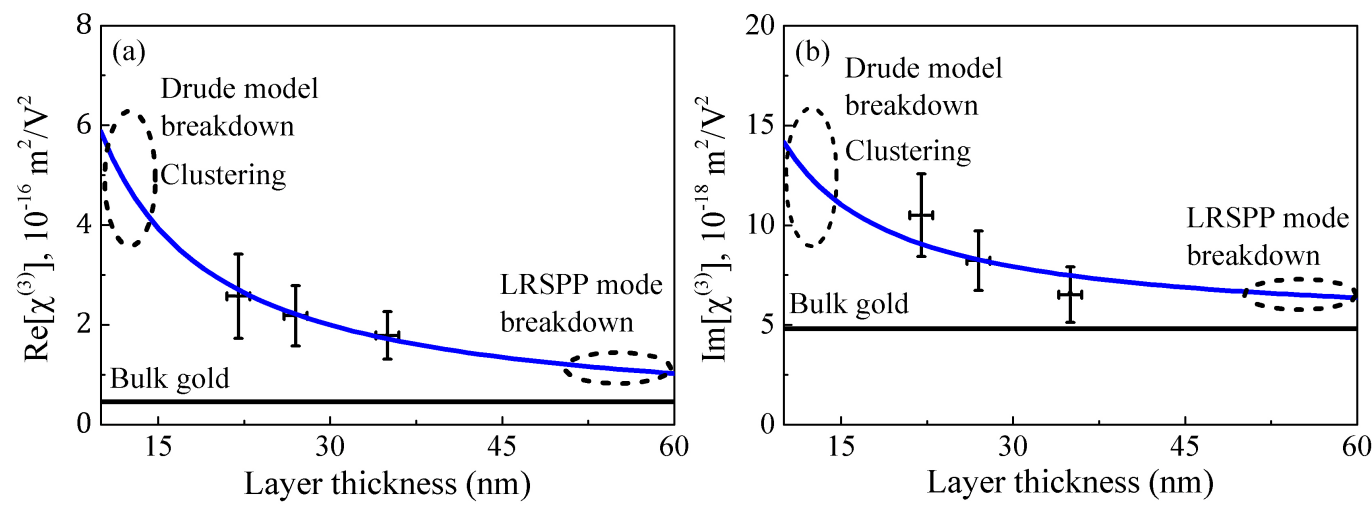

Figure 6. (a) Real part and (b) imaginary part of the third-order susceptibility for gold layers with different thicknesses. Blue curves show the nonlinear curve fit to experimental data, the black lines mark the third-order susceptibility for bulk gold.

Table 3. Third-order susceptibility of gold layers with different thicknesses.

\begin{tabular}{|c|c|c|}
\hline $\boldsymbol{t}(\mathbf{n m})$ & $\operatorname{Re}\left[\chi_{\mathrm{Au}}^{(3)}\right]\left(\mathbf{m}^{\mathbf{2}} / \mathbf{V}^{\mathbf{2}}\right)$ & $\operatorname{Im}\left[\chi_{\mathrm{Au}}^{(3)}\right]\left(\mathbf{m}^{\mathbf{2}} / \mathbf{V}^{\mathbf{2}}\right)$ \\
\hline 22 & $(2.57 \pm 0.84) \cdot 10^{-16}$ & $(10.50 \pm 2.08) \cdot 10^{-18}$ \\
\hline 27 & $(2.18 \pm 0.61) \cdot 10^{-16}$ & $(8.22 \pm 1.49) \cdot 10^{-18}$ \\
\hline 35 & $(1.79 \pm 0.48) \cdot 10^{-16}$ & $(6.51 \pm 1.38) \cdot 10^{-18}$ \\
\hline
\end{tabular}

The experimental values of the real part of $\chi_{\mathrm{Au}}^{(3)}$ are $\sim 4-5$ times higher than the real part of $\chi_{\infty}^{(3)}$ for bulk gold. And experimental values of the imaginary part of $\chi_{\mathrm{Au}}^{(3)}$ are $\sim 1.5-2$ times higher. The obtained dependencies of the third-order susceptibility for the gold layers are in accordance with previous measurements of thin gold layers using the z-scan method [25]. Figure 6 also shows the thickness limitations. The classic Drude model may be inapplicable at the layer thicknesses below 10-15 nm due to possible quantum effects [26], nonlocal effects [27], and layer clustering [28]. Conversely, the coupled LRSPP mode collapses at the layer thicknesses above 50-60 nm due to the small penetration depth of the surface electromagnetic waves in metal [20]. Therefore, the effective range of $t$ is $\sim 15-45 \mathrm{~nm}$ for experimental studies.

There are two possible reasons to explain the increase of the third-order susceptibility in gold layers with reduction of their thickness. Firstly, the effective enhancement of the third-order nonlinearity in an ultra-thin metal layer can be caused by the change in the free electrons motion due to the confinement factor. Consequently, their collisions frequency increases, and it leads to the effective increase of the dielectric permittivity of a metal layer. This, in turn, causes the 
increase of the third-order susceptibility of the metal layer. Secondly, by considering the ultra-fast electrons dynamics in metal [7], it is possible to assume that, for the electrons heated by a laser pulse, the characteristic thermalization rates with other electrons and the metal lattice change with the thickness. It may also lead to the effective enhancement of the third-order susceptibility in a metal layer [7].

The presented nonlinear optical effects in gold stripe waveguides can be exploited for further developments towards plasmonic communication components.

\section{THEORETICAL RESULTS}

\subsection{Chromatic dispersion effect}

The first and second derivatives of the real part of the LRSPP propagation constant $\beta_{1}$ and $\beta_{2}$ define the lowest-order chromatic dispersion in plasmonic waveguides. The first derivative $\beta_{1}$ is related to the group velocity $v_{\mathrm{g}}$ and group index $n_{\mathrm{g}}$ of the LRSPP mode as $\beta_{1}=d \beta / d \omega=1 / v_{\mathrm{g}}=n_{\mathrm{g}} / c$. The second derivative $\beta_{2}=d^{2} \beta / d \omega^{2}$ (also referred as the group velocity dispersion (GVD) parameter) is related to the dispersion length $L_{D}$ of the plasmonic mode as $L_{\mathrm{D}}=T_{0}^{2} /\left|\beta_{2}\right|$, where $T_{0}$ is the $1 / \mathrm{e}$-intensity value of a Gaussian input optical pulse. The normalized group velocity $v_{\mathrm{g}} / c$ and dispersion length $L_{\mathrm{D}}$ were calculated in the range 800-1550 $\mathrm{nm}$ (Fig. 7). The average value of $v_{\mathrm{g}}$ at 1064 $\mathrm{nm}$ is $\sim 67 \%$ of the vacuum speed of light. The magnitude of $\beta_{2}$ is somewhat larger $\left(\sim 10^{-4} \mathrm{ps}^{2} / \mathrm{mm}=10^{2} \mathrm{ps}^{2} / \mathrm{km}\right)$ than typical values for single-mode optical fibers $\left(\sim 10 \mathrm{ps}^{2} / \mathrm{km}\right)$ [11]. The dispersion length $L_{\mathrm{D}}$ for 3 ps pulses is much larger $(\sim 200-1000 \mathrm{~mm})$ than the physical length of the waveguides $l(\sim 1 \mathrm{~mm})$. This means that the chromatic dispersion has a small effect on the propagation of picosecond optical pulses in the plasmonic waveguide.
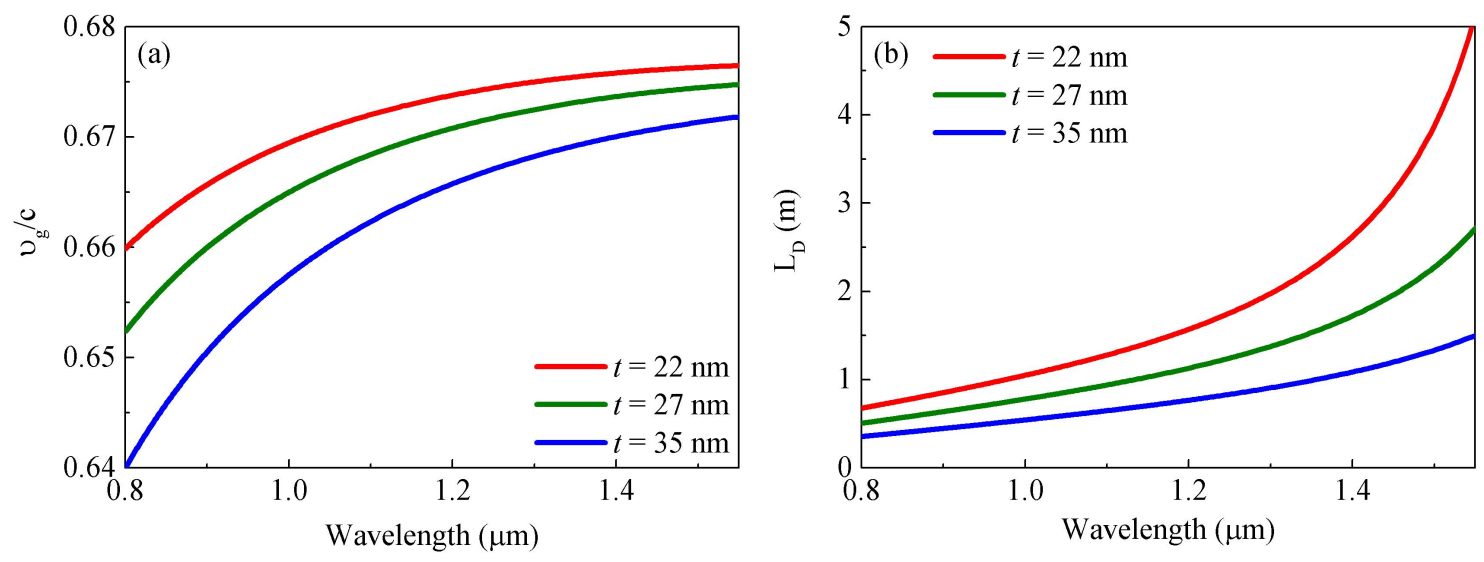

Figure 7. (a) Normalized group velocity and (b) dispersion length vs. wavelength.

\subsection{Pulse propagation equation}

The propagation dynamics of the LRSPP mode can be well described by the nonlinear Schrödinger equation [11]:

$$
\frac{\partial A}{\partial x}=i \gamma|A|^{2} A-\frac{\alpha}{2} A-\frac{\beta}{2}|A|^{2} A,
$$

where $A(x, T)$ is the complex amplitude of an optical pulse, $x$ is the propagation coordinate, $T$ is the time coordinate in the co-moving frame of the input pulse. The first and third term correspond to the self-phase modulation (SPM) and 
nonlinear absorption, respectively. The chromatic dispersion has been neglected, which is a good approximation as the dispersion length $L_{\mathrm{D}}$ for picosecond optical pulses of the plasmonic waveguide is much larger than the physical waveguide length $l$. The formal solution to Eq. (5) is $A(x, T)=\sqrt{P(x, T)} \exp \left[i \phi_{\mathrm{NL}}(x, T)\right]$, where $P(x, T)$ is the pulse power amplitude and $\phi_{\mathrm{NL}}(x, T)$ is the pulse nonlinear phase. Although this has been studied extensively in the literature, we are here dealing with the particular case where dispersion is negligible and where linear and nonlinear absorption is significant, which makes it worth to revise the results. After substitution this solution to Eq. (5), and solution of the straightforward system of differential equations, the following results are obtained for the pulse power amplitude and pulse nonlinear phase:

$$
\left\{\begin{array}{c}
P(x, T)=\frac{P_{0} e^{-\frac{T^{2}}{T_{0}^{2}}} e^{-\alpha x}}{1+\beta P_{0} e^{-\frac{T^{2}}{T_{0}^{2}}}\left(1-e^{-\alpha x}\right) / \alpha}, \\
\phi_{\mathrm{NL}}(x, T)=\frac{\gamma}{\beta} \ln \left[1+\beta P_{0} e^{\frac{T^{2}}{T_{0}^{2}}}\left(1-e^{-\alpha x}\right) / \alpha\right] .
\end{array}\right.
$$

Here the initial conditions were used: $P(0, T)=P_{0} \exp \left(-T^{2} / T_{0}^{2}\right)$ and $\phi_{\mathrm{NL}}(0, T)=0$. The solutions describe how the peak power $P(x, T)$ and phase $\phi_{\mathrm{NL}}(x, T)$ of optical pulses change in the time domain upon propagation. For practical purposes, the expression for $P(x, T)$ can be modified to obtain a formula for the average power of light at the output of the plasmonic waveguide, which is the observable and measurable quantity:

$$
\bar{P}(l)=\bar{P}(0) e^{-\alpha l}-\frac{\sqrt{\pi} \beta \bar{P}_{0}^{2}}{\sqrt{2} \alpha T_{0} f_{\text {rep }}} e^{-\alpha l} .
$$

This equation shows that the average output power $\bar{P}(l)$ from the plasmonic waveguide of the length $l$ depends nonlinearly on the average input power $\overline{P_{0}}$, and an asymptotic saturation of the function $\bar{P}(l)\left\{\overline{P_{0}}\right\}$ is expected at high values of $\overline{P_{0}}$. The nonlinear trend $\bar{P}(l)\left\{\bar{P}_{0}\right\}$ depends on the linear absorption coefficient $\alpha$, laser repetition rate $f_{\text {rep }}$, pulse duration $T_{0}$, nonlinear parameter $\beta$, and length $l$ of the waveguide. The experimental power trends for different thicknesses $t=22,27$ and $35 \mathrm{~nm}$ (Fig. 5) confirm the expected nonlinear behavior. The nonlinear term in Eq. (7) becomes important at the values of $\overline{P_{0}}$ above $0.15-0.2 \mathrm{~W}$. The possible saturation plateau is achievable at the values of $\overline{P_{0}}$ above $0.5 \mathrm{~W}$. The obtained nonlinear functions $\bar{P}(l)\left\{\overline{P_{0}}\right\}$ clearly depend on the gold thickness $t$, the saturation is achieved faster for thinner waveguides. It confirms that the gold layer thickness $t$ is a crucial parameter for $\chi^{(3)}$ of the LRSPP mode.

The second nonlinear effect concerns the LRSPP mode spectral features. The pulse power spectral density (PSD) in units $[\mathrm{W} / \mathrm{Hz}]$ is given by the squared Fourier transform of pulse amplitude $A(x, T)$ as follows:

$$
\operatorname{PSD}(x, \omega)=f_{\text {rep }}\left|\int_{-\infty}^{+\infty} A(x, T) e^{i \omega T} d T\right|^{2} .
$$

By substituting the expressions for the pulse power amplitude and phase in Eq. (26), the final formula for the PSD is obtained: 


$$
\operatorname{PSD}(l, \omega)=f_{\text {rep }}\left|\int_{-\infty}^{+\infty} \sqrt{\frac{P_{0} e^{-\frac{T^{2}}{T_{0}^{2}}} e^{-\alpha l}}{1+\beta P_{0} e^{-\frac{T^{2}}{T_{0}^{2}}} L_{\text {eff }}}} e^{i \frac{\gamma}{\beta} \ln \left[1+L_{\text {eff }} \beta P_{0} e^{-\frac{T^{2}}{T_{0}^{2}}}\right]} e^{i\left(\omega-\omega_{\mathrm{c}}\right) T} d T\right|^{2} .
$$

Here $\omega_{\mathrm{c}}=2 \pi c / \lambda$ is the carrier frequency, which corresponds to the peak wavelength $1064 \mathrm{~nm}$, and $L_{\text {eff }}=\left(1-e^{-\alpha l}\right) / \alpha$ is the effective waveguide length. The numerical calculations of Eq. (9) for the gold layer thickness $t=22 \mathrm{~nm}$ (Fig. 8) show the nonlinear spectral broadening and splitting of an input Gaussian pulse, depending on the input average power $\overline{P_{0}}$. The pulse splitting occurs at the values of $\overline{P_{0}} \sim 0.2 \mathrm{~W}$. This nonlinear effect was observed in our recent experiment [12]. The further increase of $\overline{P_{0}}$ leads to the splitting and formation of more SPM peaks.

The obtained spectral features are in accordance with the theoretical results done by Agrawal et al. for nonlinear properties of silicon waveguides [29, 30]. The principal difference here is that the plasmonic waveguide has much higher linear absorption and shorter physical length than an ordinary silicon waveguide. However, the comparable nonlinear spectral broadening can be achieved. In the case of optical fibers, the required waveguide length can reach hundreds of meters or more to receive the desired nonlinear features. In this sense a plasmonic or silicon waveguide provides the same functionality, can be integrated on-chip and used for nonlinear purposes even with very short propagation lengths.

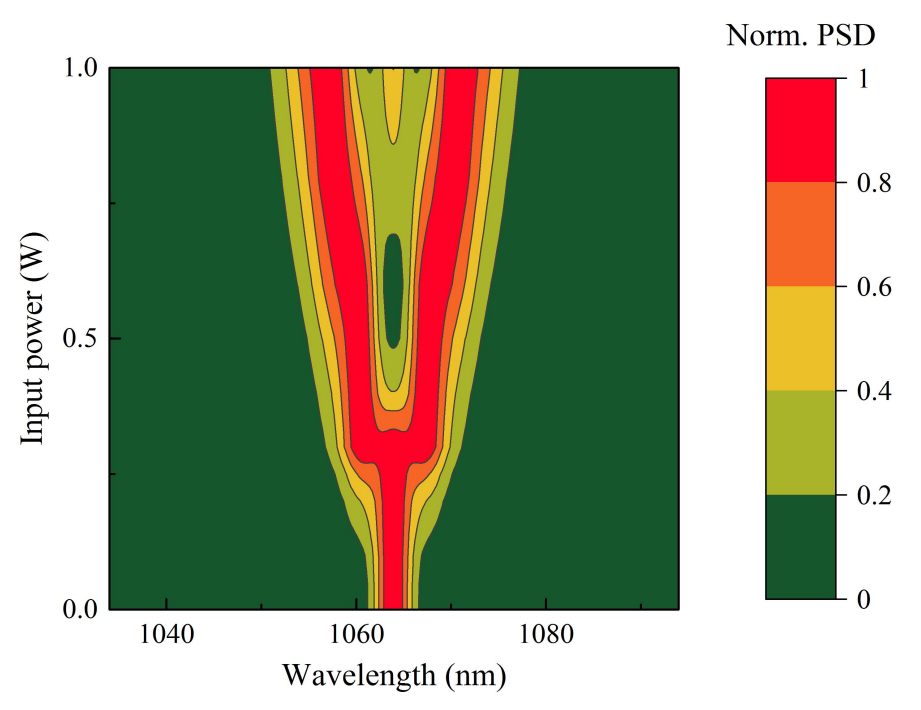

Figure 8. SPM broadening of the Gaussian pulse at $1064 \mathrm{~nm}$, using $t=22 \mathrm{~nm}$.

\section{CONCLUSION}

In this paper we have summarized the experimental and theoretical results about nonlinear effects in propagation of the LRSPP mode in gold strip waveguides. The third-order nonlinear susceptibility $\chi_{\mathrm{Au}}^{(3)}$ of the gold layers has the dominant contribution to the effective third-order susceptibility of the plasmonic mode. It was verified for three values of gold layer thickness $t=22,27$ and $35 \mathrm{~nm}$. The key assumption here is that the nonlinear properties are thickness-dependent and inversely proportional to the thickness. Thin metal layers enhance the waveguides nonlinearity and provide effective nonlinear propagation of ultra-fast picosecond optical pulses. The theoretical model, based on the solution of the nonlinear Schrödinger equation, confirmed the observed nonlinear transmission of the plasmonic modes for the average values of the input power $\overline{P_{0}}$ in the range above $0.15-0.2 \mathrm{~W}$, and for the high repetition rate $\left(f_{\text {rep }}=78 \mathrm{MHz}\right)$ picosecond pumping at $1064 \mathrm{~nm}$. In the same power range the observed SPM effect leads to the LRSPP spectral broadening and 
splitting, again in accordance with our theoretical results. The deep analogy with classical fiber-based nonlinear optics show that plasmonic waveguides are potential candidates for nonlinear optics applications on a microscopic length scale. One of the possible developments in this direction is the nonlinear analysis of higher order plasmonic modes. The current model is for the fundamental LRSPP mode, but other spatial modes can also be excited. These higher order modes will affect the dynamics by nonlinear cross-phase modulation [11]. Another development concerns the propagation of even shorter (femtosecond) pulses in the plasmonic waveguides. The chromatic dispersion effect will have a more dominant role than in the present case. The investigation of nonlinear propagation of femtosecond pulses in the plasmonic waveguides will be done elsewhere. The interaction of the nonlinear and GVD effects leads to many interesting optical phenomena in plasmonic nanostructures with their possible practical realization, for example, in optical communication systems. Our results indicate that the waveguide investigated here could have anomalous GVD at telecom C-band wavelengths, which means that solitons can be excited using femtosecond Er fiber lasers. This has interesting nonlinear applications that promise well for plasmonic systems.

\section{REFERENCES}

[1] Maradudin, A., Sambles, J. and Barnes, W., [Modern Plasmonics], Elsevier, Amsterdam, 137-187 (2014).

[2] Zenin, V., Andryieuski, A., Malureanu R., Radko, I., Volkov, V., Gramotnev, D., Lavrinenko, A. and Bozhevolnyi, S., "Boosting local field enhancement by on-chip nanofocusing and impedance-matched plasmonic antennas," Nano Lett. 15(12), 8148-8154 (2015).

[3] Krupin, O., Asiri, H., Wang, C., Tait, R. N. and Berini, P., "Biosensing using straight long-range surface plasmon waveguides," Opt. Express 21(1), 698-709 (2013).

[4] Berini, P., "Surface plasmon photodetectors and their applications," Laser Photon. Rev. 8(2), 197-220 (2014).

[5] Scalora, M., Vincenti, M., de Ceglia, D., Roppo, V., Centini, M., Akozbek, N. and Bloemer, M., "Second- and third-harmonic generation in metal-based structures," Phys. Rev. A 82(043828), 1-14 (2010).

[6] Ginzburg, P., Krasavin, A. and Zayats, A., "Cascaded second-order surface plasmon solitons due to intrinsic metal nonlinearity," New J. Phys. 15(013031), 1-13 (2013).

[7] Marini, A., Conforti, M., Della Valle, G., Lee, H. W., Tran, Tr. X., Chang, W., Schmidt, M. A., Longhi, S., Russell, P. St. J. and Biancalana F., "Ultrafast nonlinear dynamics of surface plasmon polaritons in gold nanowires due to the intrinsic nonlinearity of metals," New J. Phys. 15(013033), 1-19 (2013).

[8] Conforti, M. and Della Valle, G., "Derivation of third-order nonlinear susceptibility of thin metal films as a delayed optical response," Phys. Rev. B 85(245423), 1-4 (2012).

[9] Boyd, R. Shi, Z. and De Leon, I., "The third-order nonlinear optical susceptibility of gold," Opt. Commun. 326, 74-79 (2014).

[10] Neira, A., Olivier, N., Nasir, M., Dickson, W., Wurtz, G. and Zayats, A., "Eliminating material constraints for nonlinearity with plasmonic metamaterials," Nat. Commun., 6(7757), 1-8 (2015).

[11] Agrawal, G. P., [Nonlinear fiber Optics], 5th Edition, Elsevier, Oxford (2013).

[12] Lysenko, O., Bache, M. and Lavrinenko, A., "Third-order susceptibility of gold for ultrathin layers," Opt. Lett. 41(2), 317-320 (2016).

[13] Huck, A., Witthaut, D., Kumar, S., Soerensen, A. and Andersen, U. L., "Large optical nonlinearity of surface plasmon modes on thin gold films," Plasmonics 8(4), 1597-1605 (2013).

[14] Baron, A., Hoang, T. B., Fang, C., Mikkelsen, M. H. and Smith, D. R., "Ultrafast self-action of surfaceplasmon polaritons at an air/metal interface," Phys. Rev. B, 91(195412), 1-7 (2015).

[15] Berini, P., "Long-range surface plasmon polaritons ," Adv. Opt. Photon. 1(3), 484-588 (2009).

[16] Maradudin, A., [Light Scattering and Nanoscale Surface Roughness], Springer, New York (2007).

[17] Hunsperger, R., [Integrated Optics: Theory and Technology], Springer, Berlin, (2002).

[18] Charbonneau, R., Berini, P., Berolo, E. and Lisicka-Shrzek, E., "Experimental observation of plasmonpolariton waves supported by a thin metal film of finite width," Opt. Lett. 25(11), 844-846 (2000).

[19] Berini, P., Charbonneau, R., Lahoud, N. and Mattiussi, G., "Characterization of long-range surface-plasmonpolariton waveguides," J. Appl. Phys. 98(043109), 1-12 (2005).

[20] Maier, S., [Plasmonics: Fundamentals and Applications], Springer, New York (2007).

[21] Baron, A., Larouche, S., Gauthier, D. J. and Smith, D. R., "Scaling of the nonlinear response of the surface plasmon polariton at a metal/dielectric interface" J. Opt. Soc. Am. B 32(1), 9-14 (2015).

[22] Boyd, R., [Nonlinear Optics], Academic Press, New York (2008). 
[23] Tai, C., Wilkinson, J. S., Perney, N. M. B., Netti, M. C., Cattaneo, F., Finlayson, C. E. and Baumberg, J. J., "Determination of nonlinear refractive index in a $\mathrm{Ta}_{2} \mathrm{O}_{5}$ rib waveguide using self-phase modulation," Opt. Express 12(21), 5110-5116 (2004).

[24] Gordon, J. A. and Ziolkowski, R. W., "The design and simulated performance of a coated nano-particle laser," Opt. Express 15(5), 2622-2653 (2007).

[25] Xenogiannopoulou, E., Aloukos, P., Couris, S., Kaminska, E., Piotrowska, A. and Dynowska, E., "Third-order nonlinear optical properties of thin sputtered gold films," Opt. Commun. 275, 217-222 (2007).

[26] Xiao, Y. Qian, H. and Liu, Z., "Investigations of the optical properties of nanoscale gold films," Proc. NOMA, NM4C, 1-4 (2015).

[27] Raza, S., Bozhevolnyi, S., Wubs, M. and Mortensen, N. A., "Nonlocal optical response in metallic nanostructures," J. Phys. Condens. Matter., 27(183204), 1-17 (2015).

[28] Kreibig U. and Vollmer, M., [Optical Properties of Metal Clusters], Springer, Berlin (1995).

[29] Yin, L. and Agrawal, G. P., "Impact of two-photon absorption on self-phase modulation in silicon waveguides," Opt. Lett., 32(14), 2031-2033 (2007).

[30] Lin, Q., Painter, O. J. and Agrawal, G. P., "Nonlinear optical phenomena in silicon waveguides: Modeling and applications," Opt. Express, 15(25), 16604-16644 (2007). 